\title{
La familia Cactaceae en el Área Natural Protegida Monumento Natural Cerro del Muerto, Aguascalientes, México
}

\author{
The Cactaceae family in the Cerro del Muerto, Aguascalientes, Mexico \\ Natural Monument Protected Natural Area
}

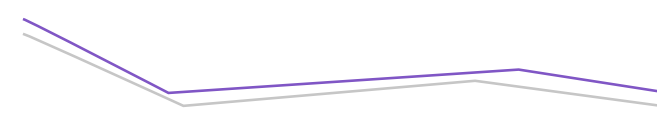

Ricardo Rivera-Martínez*凶, Gilberto-Ocampo*

Rivera-Martínez, R., \& Ocampo, G. (2018). La familia Cactaceae en el Área Natural Protegida Monumento Natural Cerro del Muerto, Aguascalientes, México. Investigación y Ciencia de la Universidad Autónoma de Aguascalientes, 26(75), 28-37.

\section{RESUMEN}

El Área Natural Protegida (ANP) Monumento Natural Cerro del Muerto se encuentra ubicado en los municipios de Aguascalientes y Jesús María, Aguascalientes, México. El plan de manejo oficial de esta ANP reporta que $8 \%$ de la flora corresponde a la familia Cactaceae; no obstante, tal documento no proporciona un listado florístico. En el borrador del plan de manejo están registrados 14 taxones de cactáceas. Ambos documentos mencionan la presencia de especies incluidas en la NOM-059SEMARNAT-2010; sin embargo, no existe evidencia física que avale esta información. En este trabajo se presenta un listado florístico conformado por 17 taxones que se encuentran en el Área Natural Protegida Monumento Natural Cerro del Muerto, de los cuales tres están incluidos dentro de la NOM059-SEMARNAT-2010, así como la referencia de los ejemplares de herbario. Además, se ofrece una clave de identificación electrónica al público en

Palabras clave: área natural protegida; Cactaceae; clave de identificación electrónica; especies sujetas a protección especial; listado florístico; plan de manejo.

Keywords: protected natural area; Cactaceae; electronic identification key; floristic list; management plan; species subject to special protection.

\section{Recibido: 10 de enero de 2018, aceptado: 11 de junio de 2018}

* Herbario, Universidad Autónoma de Aguascalientes. Av. Universidad 940, Cd. Universitaria, C. P. 20131, Aguascalientes, México. Correo electrónico: rrm.bio.a@gmail.com; gilberto.ocampo@edu.vaa.mx

四

Autor para correspondencia general, la cual permite determinar de manera sencilla la identidad de las cactáceas de esta ANP.

ABSTRACT

The Cerro del Muerto Natural Monument Protected Natural Area (PNA) is located in the municipalities of Aguascalientes and Jesus Maria within the Mexican state of Aguascalientes. The official PNA management plan reports that $8 \%$ of its flora belongs to the family Cactaceae; however, such document does not include a species list. The draft of the management plan registers 14 Cactaceae taxa. Both documents mention the presence of species registered under the NOM-059-SEMARNAT-2010; however, there is no physical evidence that supports this information. This work presents a floristic list of 17 taxa found at the Cerro del Muerto Natural Monument and Protected Natural Area, of which three are included in the NOM-059-SEMARNAT-2010, as well as the appropriate reference to the herbarium specimens as evidence that supports the provided information. In addition, and to reach a wider audience, an electronic identification key that allows an easy determination of the cacti found at this PNA is presented.

INTRODUCCIÓN

La familia Cactaceae es originaria del continente americano, se distribuye principalmente en zonas áridas y semiáridas y es una de las familias más representativas de México (Durán García \& Méndez Gonzalez, 2010). A lo largo del territorio nacional se encuentran estas plantas y se sabe que México ha sido un lugar importante de diversificación para esta familia, debido principalmente a características 


\section{InVESTIGACIÓn Y CIERCIA DE LA UחIVERSIDAD AUTÓnOMA DE RGUASCALIEOTES}

ecológicas como clima, topografía y tipo de suelos (Alanís Flores \& Velazco Macías, 2008). La familia Cactaceae cuenta con aproximadamente 1,900 especies comprendidas en 125 géneros. México tiene ca. 850 especies y es el país con mayor riqueza a nivel genérico y específico (Durán García \& Méndez González, 2010). Otros autores reconocen 913 taxones entre especies (669) y subespecies (244) agrupadas en 63 géneros. De estos, 25 géneros, 518 especies y 206 subespecies son endémicas de México (Guzmán, Arias, \& Dávila, 2007).

Los seres humanos han encontrado en esta familia un importante recurso que ha sido explotado para cubrir necesidades que van desde alimento, uso ornamental o creencias de culto religioso, hasta material para construcción (Alanís Flores \& Velazco Macías, 2008). Las especies de esta familia se han extraído de su hábitat indiscriminadamente, al grado de explotarlas tanto que hoy día es una familia amenazada (Alanís Flores \& Velazco Macías, 2008) y tiene 276 taxones protegidos dentro de la Norma Oficial Mexicana NOM-059-SEMARNAT-2010 (SEMARNAT, 2010). Por lo anterior, es muy importante tomar medidas de protección (Jiménez Sierra, 2011). Hacer un listado florístico es uno de muchos pasos que se deben seguir para lograr la conservación de especies en peligro de extinción.

El Área Natural Protegida Monumento Natural Cerro del Muerto (MNCM) se ubica en los municipios de Jesús María y Aguascalientes, en el estado de Aguascalientes, México, y fue decretada como tal el 26 de mayo de 2008 en el Periódico Oficial del estado de Aguasacalientes (Gobierno del estado de Aguascalientes, 2008).

El borrador del plan de manejo de esta área natural protegida describe a grandes rasgos el tipo de vegetación del lugar y enlista 14 especies de la familia Cactaceae (Gobierno del estado de Aguascalientes, inédito). Por otra parte, el plan de manejo oficial solo menciona que $8 \%$ de la flora total del ANP pertenece a la familia Cactaceae; sin embargo, en este documento se omiten cuáles son las especies que componen ese porcentaje (Secretaría de Medio Ambiente del estado de Aguascalientes, 2015). Ambos documentos, el borrador del plan de manejo y el plan de manejo oficial del Gobierno del estado de Aguascalientes (inédito, 2008) reportan dos especies de plantas que están en la categoría sujeta a protección especial dentro de la NOM-059-SEMARNAT-2010 (SEMARNAT,
2010), una de ellas es Ferocactus histrix (DC.) G.E. Linds., miembro de la familia Cactaceae. Hasta donde se sabe, no se encuentran disponibles en ningún herbario ejemplares que avalen el listado florístico de esta familia que se incluyen en el borrador del plan de manejo y que se mencionan como cactáceas en el plan de manejo mismo. Las especies que se enlistan en el borrador del plan de manejo son las siguientes:

Ferocactus histrix (DC.) G.E. Linds.

Mammillaria uncinata Zucc. ex Pfeiff.

Myrtillocactus geometrizans (Mart. ex Pfeiff.) Console. Neolloydia conoidea (DC.) Britton \& Rose.

Opuntia cantabrigensis Lynch.

Opuntia chavena Griffiths.

Opuntia durangensis Britton \& Rose.

Opuntia ficus-indica (L.) Mill.

Opuntia grahamii Engelm.

Opuntia imbricata (Haw.) DC.

Opuntia rastrera F.A.C. Weber.

Opuntia robusta J. C. Wendl.

Opuntia stenopetala Engelm.

Opuntia streptacantha Lem.

De acuerdo con el borrador del plan de manejo, se tienen registrados cinco géneros en el MNCM y Opuntia es el que muestra mayor riqueza de especies, seguido en menor proporción por Ferocactus, Mammillaria, Myrtillocactus y Neolloydia, en donde hay registro de una especie para cada uno de esos géneros, como se puede observar en la figura 1.

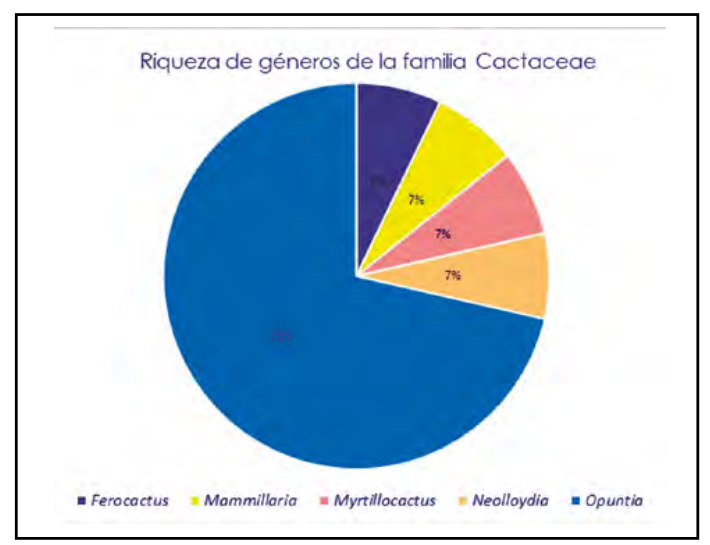

Figura 1. Riqueza de especies por género de la familia Cactaceae presentes en el Área Natural Protegida Monumento Natural Cerro del Muerto.

Elaboración propia con información recabada del borrador del plan de manejo del Área Natural Protegida Monumento Natural Cerro del Muerto (Gobierno del estado de Aguascalientes, inédito). 


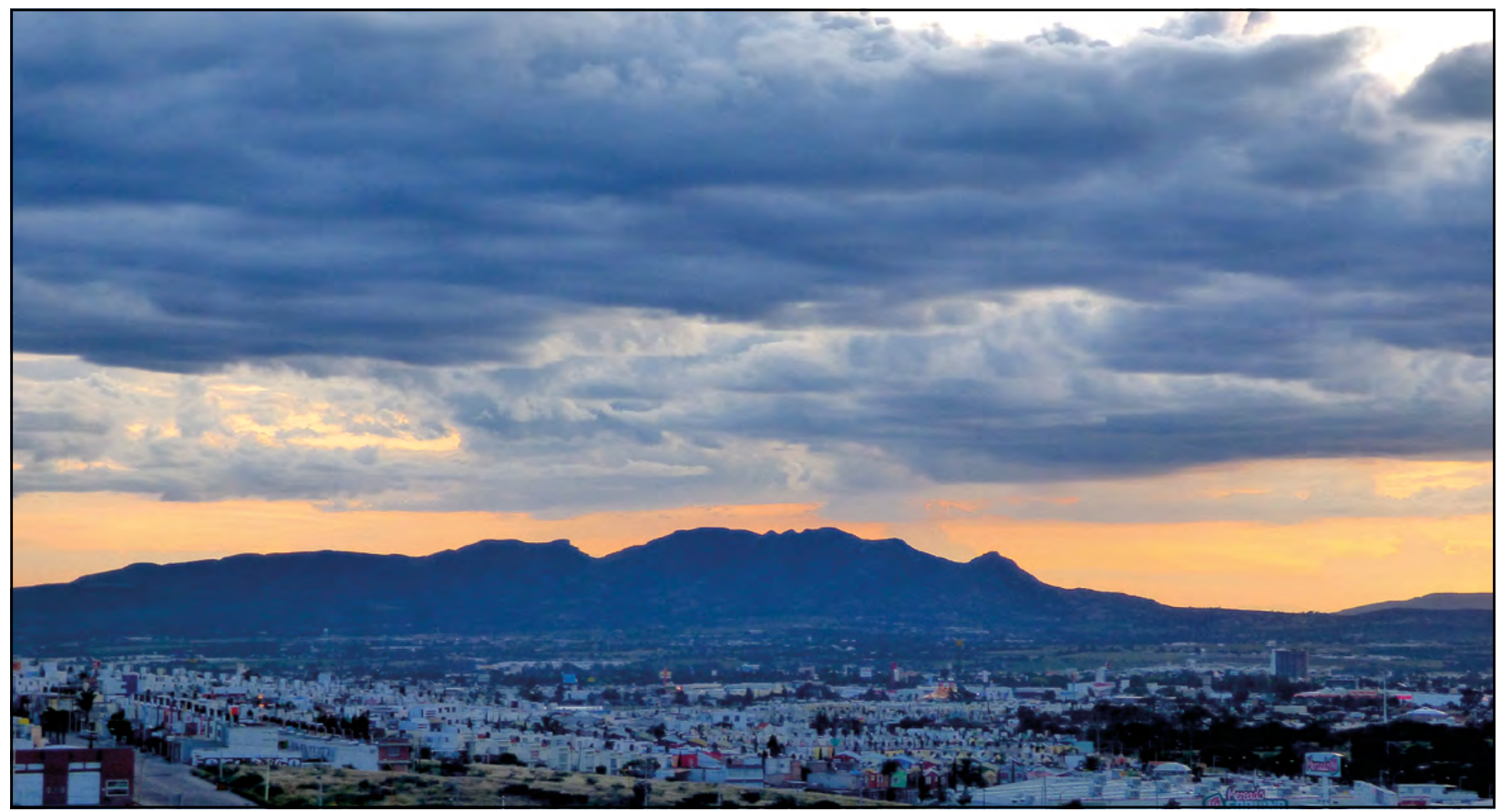

Figura 2. Vista panorámica del Área Natural Protegida Monumento Natural Cerro del Muerto.

Fotografía tomada por Luisalvaz (2014).

Debido a la falta de documentación sobre la presencia de especies de Cactaceae en esta ANP, el presente estudio buscó corroborar la información ofrecida en los documentos oficiales (borrador y plan de manejo). La hipótesis de trabajo fue que se deberían de encontrar en el MNCM las especies reportadas en los documentos mencionados. El estudio se efectuó a través de salidas a campo y de la obtención de ejemplares de herbario, los cuales representan una evidencia clara sobre la presencia de la familia Cactaceae en el MNCM.

\section{MATERIALES Y MÉTODOS}

Según información recabada del plan de manejo oficial del MNCM (Secretaría de Medio Ambiente del estado de Aguascalientes, 2015), esta ANP se encuentra ubicada en los municipios de Jesús María y Aguascalientes, entre los paralelos $21^{\circ} 47^{\prime} 47.6^{\prime \prime}$ y $21^{\circ}$ 53' 26.4' ' norte y los meridianos $102^{\circ} 22^{\prime} 49.9^{\prime \prime}$ y $102^{\circ}$ 28' 52.3' 'oeste. El MNCM se compone de un macizo montañoso cuyo eje corre de suroeste a noreste, así como de una región de planicie, que tiene un área total de 5,862.03 ha (figura 2). La elevación se encuentra entre los 1,842 y los 2,440 m.s.n.m. La geología del lugar está compuesta primordialmente por rocas ígneas extrusivas del Cenozoico, mientras que los suelos más predominantes son del tipo
Feozem. El clima de esta ANP es mayoritariamente semiseco templado con lluvias en verano y escasas a lo largo del año (BSI kw), así como templado con verano cálido y lluvias en verano (C(w0)).

En las partes más bajas y en laderas se encuentran zonas con pastizales, matorral xerófilo y matorral subtropical, mientras que en las partes más altas se observa el bosque de encino. Para el MNCM se encuentran reportadas la presencia de 182 especies de plantas pertenecientes a 122 géneros y 43 familias. De estas últimas las más abundantes son Asteraceae (24\%), Poaceae (21\%), Cactaceae (8\%) y Fabaceae (7\%). La riqueza de especies vegetales representa el $8.5 \%$ del total de plantas reportadas para el estado de Aguascalientes, la cual se estima en 2128 (Martínez-Ramírez, Siqueiros-Delgado, De la Cerda Lemus, \& García Regalado, inédito). Cabe destacar que en el MNCM se realizan actividades recreativas y turismo de bajo impacto (Secretaría de Medio Ambiente del estado de Aguascalientes, 2015).

Para muestrear el MNCM se llevaron a cabo 11 visitas en el periodo comprendido desde enero hasta octubre de 2016, mediante transectos desde puntos estratégicos ubicados en diversas partes del MCNM, dirigidos hacia el eje del macizo montañoso (figura 3). 


\section{IIVESTIGAGIÓn Y CUERCIA DE LA UחIVERSIDAD AUTÓnOMH

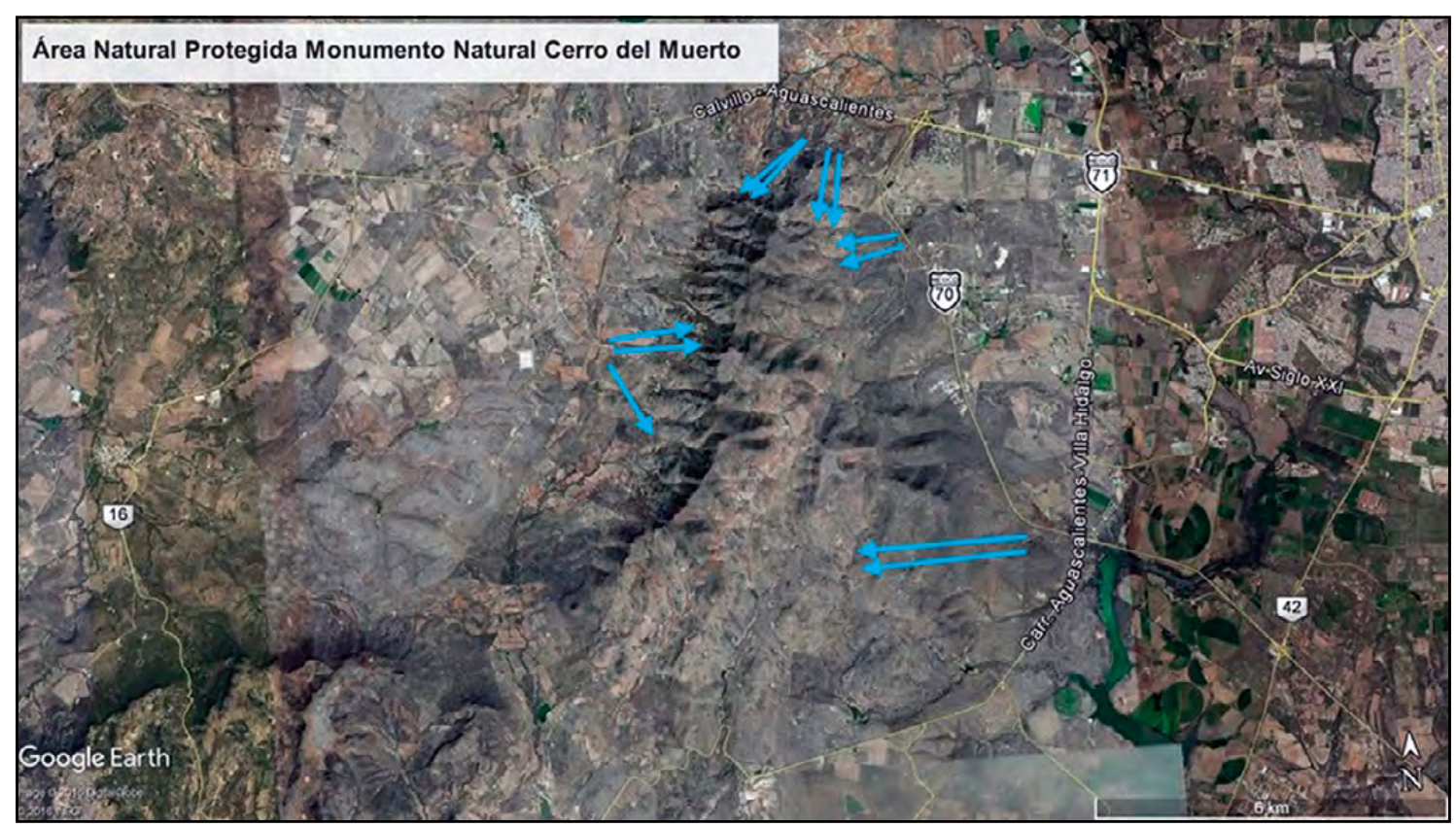

Figura 3. Transectos realizados en el Área Natural Protegida Monumento Natural Cerro del Muerto. La dirección de la flecha indica su orientación.

Imagen satelital tomada de Google Earth (2016).

Las recolecciones se efectuaron a medida que se hacía el recorrido siguiendo la dirección del transecto planeado. Se siguió el método propuesto por Sánchez-González \& González Ledesma (2007), que sugiere seleccionar ejemplares saludables y procurar que cuenten con material reproductivo (flor y/o fruto). También se recopilaron ejemplares sin estructuras reproductivas, ya que algunas especies pueden identificarse utilizando caracteres vegetativos, como forma y color de tallo, tamaño y número de espinas, tamaño de la planta, entre otros.

Para la recolección y herborización de los ejemplares de cactáceas se siguieron algunas de las recomendaciones propuestas por De Grooł (2011) y Sánchez Mejorada (1986). Entre ellas destacan la realización de cortes estratégicos en la planta para conservar el ejemplar de la manera más natural. Es importante considerar la conservación de espinas y areolas (lunares de donde surgen las espinas), llevar a cabo la eliminación parcial del tejido del tallo que es rico en agua (parénquima) y el uso de sal para facilitar la deshidratación del ejemplar para prevenir infecciones fúngicas, entre otras.
Una vez deshidratados los ejemplares se procedió a efectuar la identificación de cada organismo recolectado. Para ello se utilizaron las claves de Bravo-Hollis (1978), De la Cerda-Lemus (1999), González-Durán, Riojas-López, \& Arreola-Nava (2001) y Scheinvar (2004), así como artículos y documentos adicionales (Sánchez Macías, 2016).

La clave de identificación electrónica se realizó a través del software $\mathrm{Xper}^{2}$ (Ung, Dubus, ZaragüetaBagils, \& Vignes-Lebbe, 2010), sistema de gestión para el almacenamiento, edición y análisis de datos descriptivos (cualitativos y cuantitativos). Una vez obtenidos los datos y fotografías se eligieron los caracteres y estados de carácter para identificar las especies que se encuentran en el MNCM. La base de datos creada en $X p^{2}{ }^{2}$ se exportó en formato SDD y se incorporó al sistema en línea $X p^{3}{ }^{3}$ (LIS, s. f. b; Vignes-Lebbe, Chesselet, \& Diep Thi, 2016). Cabe mencionar que este recurso está dirigido a la población en general, por lo que se evitó el uso de tecnicismos y se procuró manejar un vocabulario más coloquial.

RESULTADOS

Los taxones encontrados en la zona de estudio son los siguientes y pueden observarse en las figuras 4 a 6 : 
Coryphantha ottonis (Pfeiff.) Lem.

Echinocereus sp.

Ferocactus histrix (DC.) G.E. Linds.

Mammillaria bombycina Quehl subsp. bombycina

Mammillaria bombycina subsp. perezdelarosae Bravo \& Scheinvar.

Mammillaria uncinata Zucc. ex Pfeiff.

Opuntia durangensis Britton \& Rose

Opuntia hyptiacantha F.A.C. Weber

Opuntia imbricata (Haw.) DC.
Opuntia jaliscana Bravo

Opuntia joconostle F.A.C. Weber ex Diguet

Opuntia lasiacantha Pfeiff.

Opuntia rastrera F.A.C. Weber

Opuntia robusta J. C. Wendl.

Opuntia streptacantha Lem.

Opuntia tomentosa Salm-Dyck

Stenocactus heteracanthus (Muehlenpf.) A. Berger ex A.W. Hill

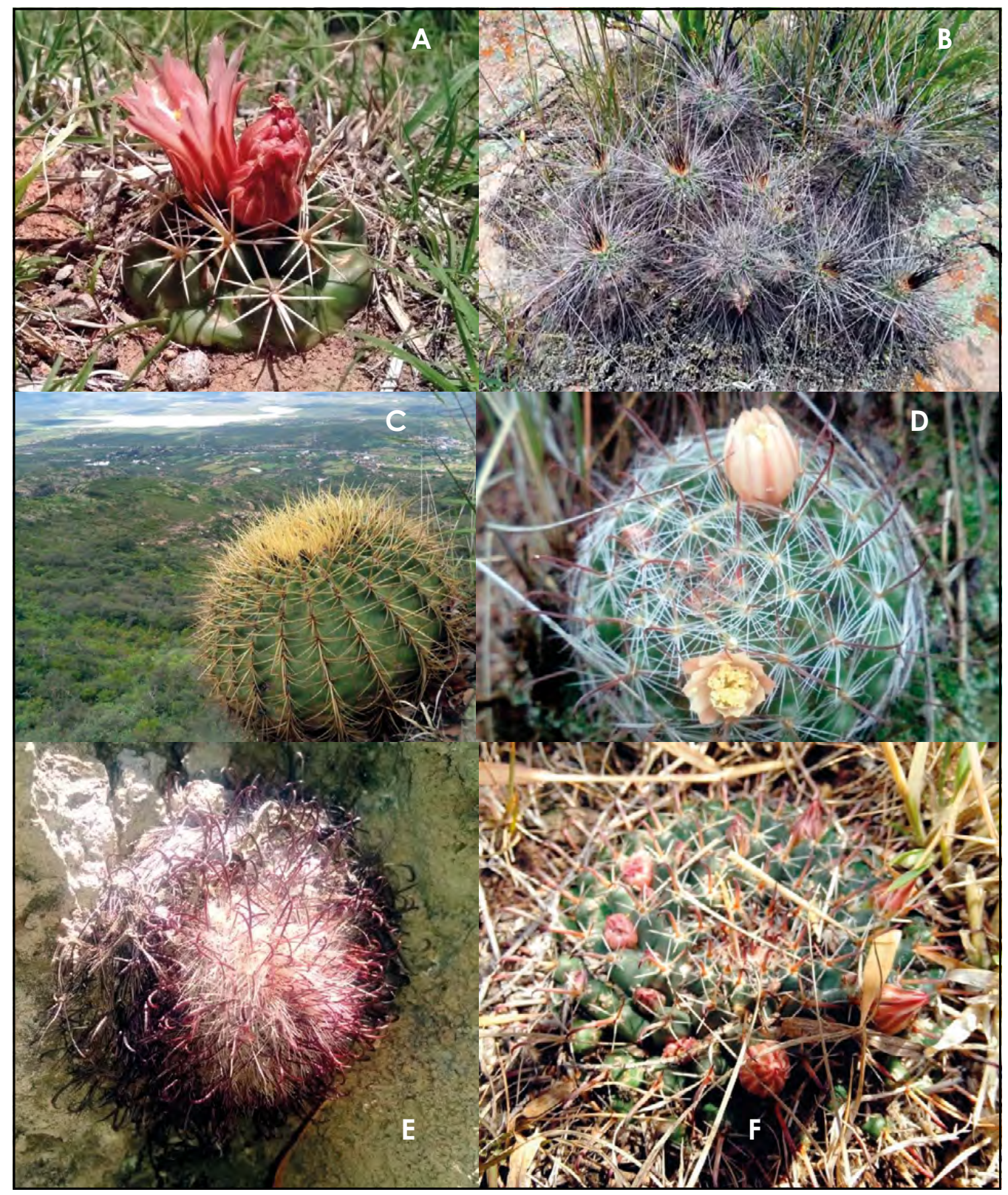

Figura 4. Cactáceas del Área Natural Protegida Monumento Natural Cerro del Muerto. A) C. ottonis, B) E. sp., C) F. histrix, D) M. bombycina subsp. bombycina, E) M. bombycina subsp. perezdelarosae, F) M. uncinata.

Fotografías del equipo de investigación. 


\section{InVESTIGACIÓn Y CIERCIA DE LA UחIVERSIDAD AUTÓnOMA DE RGUASCALIEחTES}

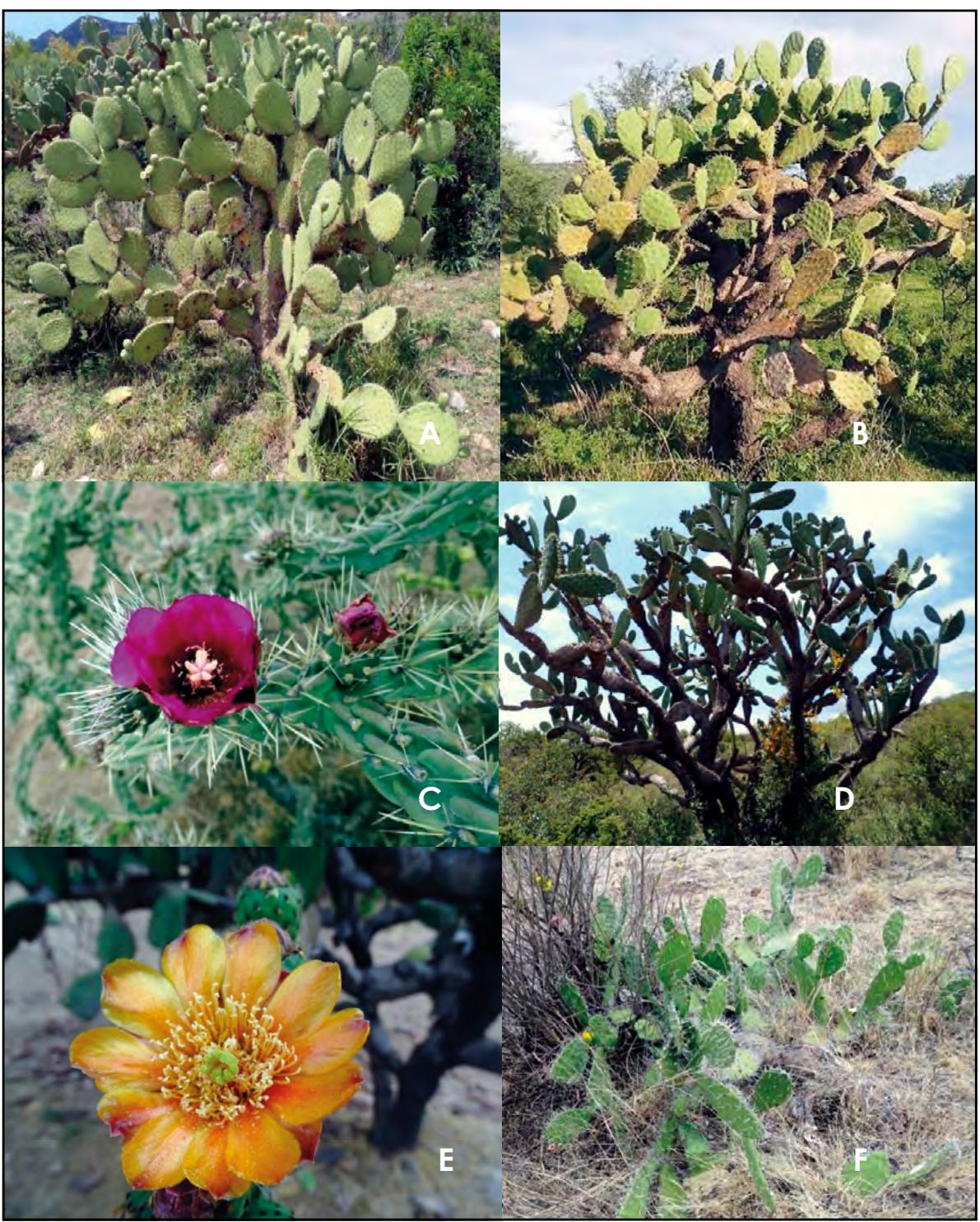

Figura 5. Cactáceas del Área Natural Protegida Monumento Natural Cerro del Muerto. A) O. durangensis, B) O. hyptiacantha, C) O. imbricata, D) O. jaliscana, E) O. lasiacantha (flor), F) O. rastrera.

Fotografías del equipo de investigación.

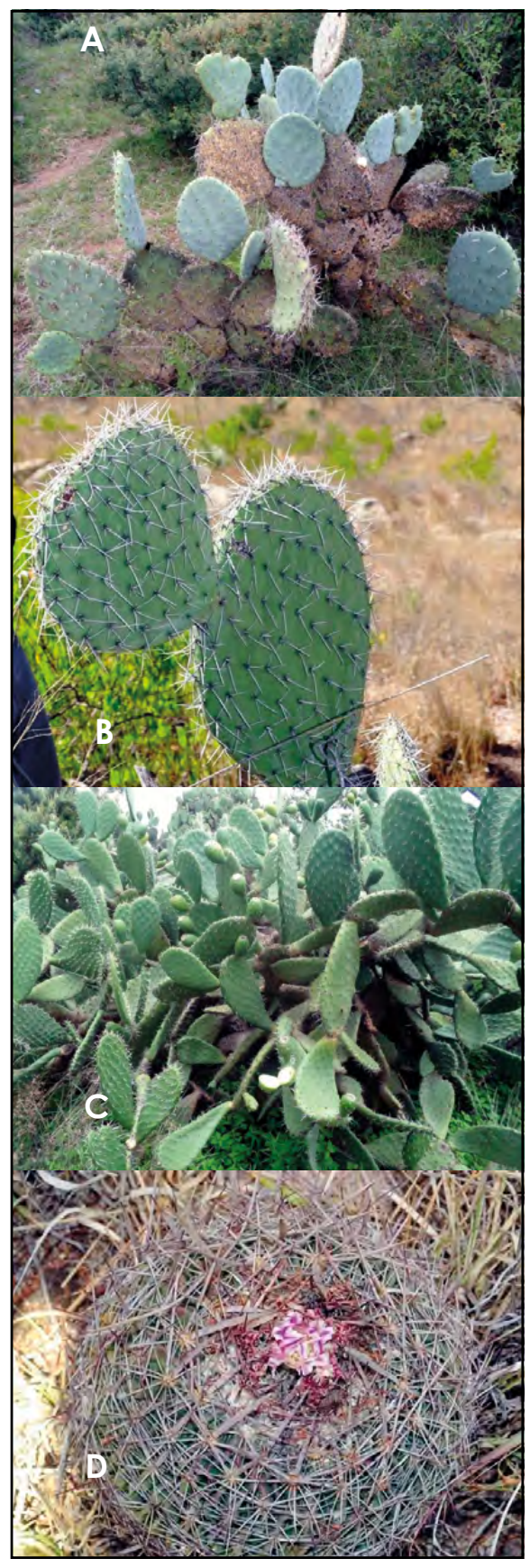

Figura 6. Cactáceas del Área Natural Protegida Monumento Natural Cerro del Muerto. A) O. robusta, B) O. streptacantha, C) O. tomentosa, D) S. heteracanthus. Fotografías del equipo de investigación. 


\section{LA UNIVERSIDAD AUTÓกOMA DE RGUASCALIERTES}

La figura 7 muestra la riqueza genérica reportada en este estudio. El género Opuntia es el que presenta más especies, seguido por Mammillaria en menor proporción. Por último, los géneros Coryphanta, Echinocereus, Ferocactus y Stenocactus presentan solo una especie por género.

En la tabla 1 se muestra la comparación entre el listado resultante del presente trabajo y el listado del borrador del plan de manejo con énfasis en sus diferencias.

Los ejemplares de herbario que resultaron de este estudio fueron depositados en el herbario de la

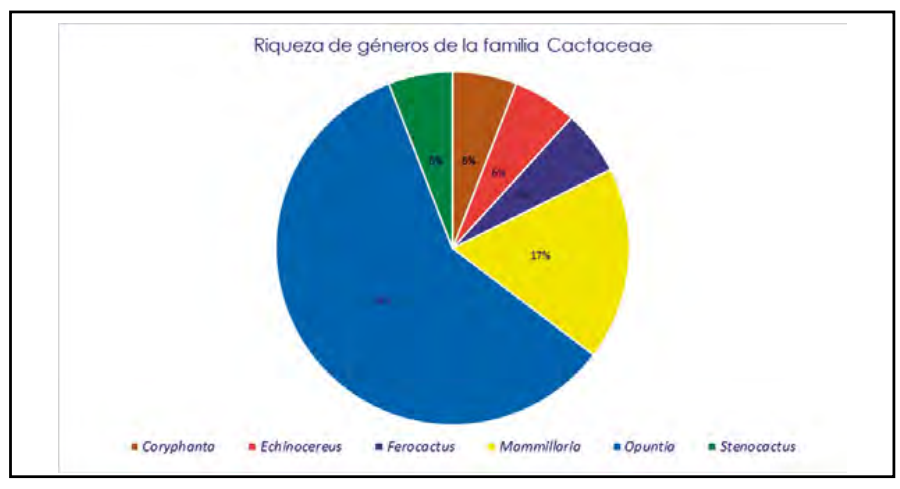

Figura 7. Porcentaje que representa la riqueza de cada uno de los géneros de la familia Cactaceae presentes en el Área Natural Protegida Monumento Natural Cerro del Muerto.

Elaboración propia.

Tabla 1

Comparación del listado florístico de este estudio con el del borrador del plan de manejo del Área Natural Protegida Monumento Natural Cerro del Muerto

\begin{tabular}{|c|c|}
\hline Especies documentadas en este estudio & $\begin{array}{l}\text { Especies enlistadas en el borrador del plan de manejo del } \\
\text { MNCM }\end{array}$ \\
\hline \multicolumn{2}{|l|}{ Coryphantha ottonis (Pfeiff.) Lem. } \\
\hline \multicolumn{2}{|l|}{ Echinocereus sp. } \\
\hline Ferocactus histrix (DC.) G.E. Linds. & Ferocactus histrix (DC.) G.E. Linds. \\
\hline \multicolumn{2}{|l|}{ Mammillaria bombycina Quehl subsp. bombycina } \\
\hline \multicolumn{2}{|c|}{$\begin{array}{l}\text { Mammillaria bombycina subsp. perezdelarosae (Bravo } \\
\text { \&Scheinvar) D.R. Hunt }\end{array}$} \\
\hline \multirow[t]{4}{*}{ Mammillaria uncinata Zucc. ex Pfeiff. } & Mammillaria uncinata Zucc. ex Pfeiff. \\
\hline & Myrtillocactus geometrizans (Mart. ex Pfeiff.) Console \\
\hline & Neolloydia conoidea (DC.) Britton \& Rose \\
\hline & $\begin{array}{l}\text { Opuntia cantabrigensis Lynch (= Opuntia engelmannii Salm- } \\
\text { Dyck ex Engelm.) }\end{array}$ \\
\hline Opuntia durangensis Britton \& Rose & $\begin{array}{l}\text { Opuntia grahamii Engelm. (= Grusonia grahamii (Engelm.) } \\
\text { H. Rob.) }\end{array}$ \\
\hline Opuntia hyptiacantha F.A.C. Weber & $\begin{array}{l}\text { Opuntia chavena Griffiths (= Opuntia hyptiacantha F.A.C. } \\
\text { Weber) }\end{array}$ \\
\hline
\end{tabular}

Opuntia jaliscana Bravo

Opuntia joconostle F.A.C. Weber ex Diguet

Opuntia imbricata (Haw.) DC. Opuntia imbricata (Haw.) DC.

Opuntia lasiacantha Pfeiff.

Opuntia rastrera F.A.C. Weber Opuntia rastrera F.A.C. Weber

Opuntia robusta J.C. Wendl. Opuntia robusta J.C. Wendl.

Opuntia streptacantha Lem. Opuntia streptacantha Lem.

Opuntia tomentosa Salm.Dyck

Stenocactus heteracanthus (Muehlenpf.) A. Berger ex

A.W. Hill

Nota: Las celdas en azul muestran las especies en común. Los nombres entre paréntesis y precedidos por un signo "=" indican el nombre aceptado.

Elaboración propia. 
Tabla 2

Caracteres y estados de carácter utilizados para la elaboración de la clave electrónica de identificación en Xper²

\begin{tabular}{ll}
\hline & \multicolumn{1}{c}{ Carácter } \\
\hline Tallo articulado & Ausente-Presente \\
Tallo aplanado & Ausente-Presente \\
Forma del tallo & Globoso-Cilíndrico-Aplanado \\
Tallo con costillas & Ausente-Presente \\
Forma de las costillas & Onduladas-No onduladas-No aplica \\
Tallo formado por tubérculos en forma de chichones & Ausente-Presente \\
(mamilas) & \\
Surco en los tubérculos & Ausente-Presente-No aplica \\
\hline
\end{tabular}

Nota: Elaboración propia.

Universidad Autónoma de Aguascalientes (HUAA). En algunos casos se obtuvieron ejemplares duplicados, por lo que serán enviados como intercambio a otros herbarios nacionales.

Los caracteres y estados de carácter que se utilizaron para la creación de la clave de identificación electrónica se muestran en la tabla 2. La clave electrónica de identificación se puede consultar en el sitio de Laboratoire Informatique \& Systematique (LIS, s. f. a).

\section{DISCUSIÓN}

El listado florístico que resultó de este estudio concuerda parcialmente con el reportado en el borrador del plan de manejo del MNCM, mismo que enlista 14 especies de cactáceas, de las cuales ocho están presentes en este estudio, mientras que las otras seis no fueron encontradas en los recorridos efectuados. Es posible que las especies reportadas en el borrador del plan se encuentren en sitios que no fueron visitados en este estudio; sin embargo, el borrador mencionado no proporciona datos acerca de ejemplares de herbario de respaldo, ni información acerca de su georreferenciación, por lo que los registros ofrecidos en ese documento son dudosos.

Los resultados de este estudio muestran la presencia de seis géneros de cactáceas, mientras que el borrador del plan de manejo reporta cinco (figuras 1 y 7). De ellos únicamente Ferocactus, Mammillaria y Opuntia coinciden en ambos estudios y concuerdan en que el género Opuntia es el que tiene mayor número de especies en el área.

El plan de manejo oficial del MNCM menciona que dentro del área hay dos plantas protegidas en la NOM-059-SEMARNAT-2010 (SEMARNAT, 2010). Una de estas plantas es Ferocactus histrix, la cual se encuentra en la categoría sujeta a protección especial. Como resultado del presente trabajo se cuenta con un ejemplar de herbario que avala la presencia de esta especie dentro del área. Por otra parte, en este estudio se encontraron dos taxones más que están presentes en esta norma y que están bajo la categoría sujeta a protección especial: Mammillaria bombycina subsp. bombycina y M. bombycina subsp. perezdelarosae. Cabe destacar que en tal lista ambas subespecies están registradas a nivel especie, aunque otros trabajos consideran a M. perezdelarosae como una subespecie de $M$. bombycina (CONABIO, 2009), criterio que se sigue en este trabajo.

Se observó que los lugares más alejados a terracerías o senderos donde la gente acude a realizar actividades recreativas tienen una riqueza mayor de especies de la familia Cactaceae. A su vez, los lugares más cercanos a áreas recreativas tienen poca representatividad de especies de cactáceas, con excepción del género Opuntia (mejor conocido como nopal) que se encuentra prácticamente en toda el área. Por ejemplo, el lugar conocido localmente como Cerro El Picacho, ubicado al norte del polígono que delimita el MNCM, presenta una perturbación mayor, en donde las únicas especies de Cactaceae que se pudieron observar fueron las del género Opuntia.

Una de las amenazas que tiene la familia Cactaceae dentro del MNCM es la extracción ilegal de estas plantas de su hábitat. Se observó directamente en campo que la gente que acude a esta zona extrae cactáceas sin ninguna restricción. Esto 
IחVESTIGAGIÓn Y GIEnCIA DE LA UNIVERSIDAD AUTÓNOMA constituye un riesgo grande ya que las poblaciones se ven mermadas y disminuyen el número de individuos y capacidad de reproducción y persistencia de las especies en esta ANP.

Las claves de identificación electrónicas son una herramienta relativamente nueva, en donde la información sobre biodiversidad puede compartirse de una manera más práctica, tanto con los sectores académicos y científicos, como con la sociedad en general (Ung et al., 2010). Actualmente esta clave se encuentra disponible a través del sistema $\mathrm{Xper}^{3}$ en el sitio de LIS (s. f. a). El objetivo de ofrecer este recurso es que la población de la entidad que acude a esta área conozca y se relacione con la familia Cactaceae para generar conciencia en cuanto al cuidado y conservación de estas plantas.

\section{CONCLUSIONES}

La familia Cactaceae está presente en el MNCM y ahora cuenta con la documentación pertinente (ejemplares de herbario) que avala esta información. De los taxones registrados, tres están protegidos bajo la NOM-059-SEMARNAT-2010 (SEMARNAT, 2010), lo que le brinda mayor valor biológico y de conservación a esta área.

El listado florístico que resulta de este estudio concuerda parcialmente con el presente en el borrador del plan de manejo, lo que lleva a rechazar la hipótesis establecida para este trabajo. Sin embargo, la información aquí obtenida pretende contribuir al plan de manejo y a la conservación de la biodiversidad del MNCM. Se espera que la información generada en este estudio sea considerada y retomada por las dependencias gubernamentales competentes (e.g., Secretaría de Sustentabilidad, Medio Ambiente y Agua del gobierno del estado de Aguascalientes) para ser incluida en el plan de manejo oficial del MNCM.

Se recomienda continuar y alentar el desarrollo de estudios relacionados con la familia Cactaceae en el MNCM. En particular, será importante contar con información sobre aspectos ecológicos y poblacionales de las especies, para comprender de manera integral los factores que puedan impactar las cactáceas de la zona de estudio.

La clave de identificación electrónica es una herramienta que promoverá la difusión de la información generada en este estudio hacia la sociedad en general. Se tiene como plan a corto plazo gestionar el apoyo de las dependencias encargadas del ANP para ofrecer medios informativos en forma de gacetas o folletos, divulgar esta información y contribuir con la preservación de las cactáceas dentro del MNCM.

\section{Agradecimientos}

Los autores desean agradecer al Departamento de Biología y al herbario de la Universidad Autónoma de Aguascalientes (HUAA) por brindar las facilidades necesarias para desarrollar este trabajo.

Desean, asimismo, externar un agradecimiento al Biól. Julio Martínez, encargado del HUAA, por apoyar este estudio con sus conocimientos y disposición para resolver dudas.

También agradecen a la SEMARNAT por haber otorgado el permiso con número de oficio SGPA/ DGVS/01149/16 para efectuar las recolecciones de plantas de la familia Cactaceae dentro del marco legal. 


\section{REFERENCIAS}

- Alanís Flores, G. J., \& Velazco Macías, C. G. (2008). Importancia de las cactáceas como recurso natural en el noreste de México. CIENCIA-UANL, $11(1), 5-11$.

- Bravo-Hollis, H. (1978). Las cactáceas de México. Volumen 1 (2a. ed.). México: Universidad Nacional Autónoma de México.

- Comisión Nacional para el Conocimiento y Uso de la Biodiversidad. (2009). Catálogo taxonómico de especies de México. México: Autor.

- De Groot, S. J. (2011). Collecting and Processing cacti into herbarium specimens, using ethanol and other methods. Systematic Botany, 36(4), 981-989.

- De la Cerda-Lemus, M. (1999). Cactáceas de Aguascalientes (2a. ed.). Aguascalientes, México: Universidad Autónoma de Aguascalientes.

- Durán García, R., \& Méndez González, M. E. (2010). Biodiversidad: Cactaceas. En Biodiversidad y Desarrollo Humano en Yucatán (pp. 191-192). Mérida, Yucatan: CICY/ PPD-FMAM/CONABIO/SEDUMA.

- Gobierno del estado de Aguascalientes. (inédito). Borrador del programa de manejo. Recuperado de http://www. aguascalientes.gob.mx/Imae/Pdf/PROGRAMA_MANEJO_ CERRO_MUERTO.pdf

- Gobierno del estado de Aguascalientes. (26 de mayo de 2008). Decreto por el que se declara área natural protegida en la categoría de Monumento Naural al Cerro del Muerto. Periodico Oficial del estado de Aguascalientes, 5-11.

- González-Durán, A., Riojas-López, M. E., \& Arreola-Nava, H. J. (2001). El género Opuntia en Jalisco. Guía de campo. Jalisco, México: Universidad de Guadalajara-Comision Nacional para el Conocimiento y Uso de la Biodiversidad.

- Google Earth. (2016). Imagen satelital del Área Natural Protegida Monumento Natural Cerro del Muerto [Fotografía]. Recuperada el 11 de noviembre de 2017, de https://earth. google.com

- Guzmán, U., Arias, S., \& Dávila, P. (2007). Catálogo de cactáceas mexicanas. D. F., México: UNAM-CONABIO.

- Jiménez Sierra, C. L. (2011). Las cactáceas mexicanas y los riesgos que enfrentan. Revista Digital Universitaria, 12(1), 1-23.

- Laboratoire Informatique \& Systematique (s. f. a). Cactáceas del Cerro del Muerto (Ags., México) [Claves electrónicas de identificación]. Recuperadas de http://www.xper3.fr/xper3GeneratedFiles/publish/ identification/343692455781091785/mkey.html
- Laboratoire Informatique \& Systématique (s. f. b). Xper3. The biodiversity collaborative management platform [Portal electrónico]. París: Autor. Recuperada de www.xper3.fr

- Luisalvaz. (2014). Atardecer en el Cerro del Muerto [Fotografía]. Recuperada de https://es.wikipedia.org

- Martínez-Ramírez, J., Siqueiros Delgado, M. E., De la Cerda Lemus, M., \& García Regalado, G. (inédito). $2^{\circ}$ Listado florístico del estado de Aguascalientes.

- Sánchez-González, A., \& González Ledesma, M. (2007). Técnicas de recolecta de plantas y herborización. En A. Contreras-Ramos, C. Cuevas Cardona, I. Goyenechea, \& U. Iturbe (Eds.), La sistemática, base del conocimiento de la biodiversidad (pp. 123-133). Pachuca, Hidalgo: Universidad Autonóma del Estado de Hidalgo.

- Sánchez Macías, I. G. (2016). Actualización taxonómica de la familia Cactaceae en el estado de Aguascalientes. Aguascalientes, México: Universidad Autónoma de Aguascalientes.

- Sánchez Mejorada, H. (1986). Suculentas. En A. Lot \& F. Chiang (Comps.), Manual de Herbario. Administración y manejo de colecciones, técnicas de recolección y preparación de ejemplares botánicos (pp. 103-111). D. F., México: Consejo Nacional de la Flora de México, A. C.

- Scheinvar, L. (2004). Flora cactológica del estado de Queretaro: Diversidad y riqueza. Queretaro, México: Fondo de Cultura Económica.

- Secretaría de Medio Ambiente del estado de Aguascalientes. (2015). Programa de Manejo del Área Natural Protegida Monumento Natural Cerro del Muerto. Periodico Oficial Del Estado de Aguascalientes. Aguascalientes, México.

- Secretaría de Medio Ambiente y Recursos Naturales. (30 de diciembre de 2010). NORMA OFICIAL MEXICANA NOM-059SEMARNAT-2010, Protección ambiental-Especies nativas de México de flora y fauna silvestres-Categorias de riesgo y especificaciones para su inclusión, exclusión o cambioLista de especies en riesgo. Diario Oficial de la Federación, Segunda sección, 1-78.

- Ung, V., Dubus, G., Zaragüeta-Bagils, R., \& Vignes-Lebbe, R. (2010). Xper2: Introducing e-taxonomy. Bioinformatics, 26(5), 703-704.

- Vignes-Lebbe, R., Chesselet, P., \& Diep Thi, M. H. (2016). Xper3: new tools for collaborating, training and transmitting knowledge on botanical phenotypes. En N. R. Rakotoarisoa, S. Blackmore, \& Riera, B. (Eds.), Proceedings of the UNESCO International Conference, Botanists of the twenty-first century: roles, challenges and opportunities (pp. 228-239). París: UNESCO. 